\title{
HonIoT: Arquitetura de Honeynet com Controle de Propagação de Malwares para Dispositivos de IoT
}

\author{
Douglas B. de Godoy ${ }^{1}$, Jaime F. Souza ${ }^{1}$, Emerson R. A. Barea ${ }^{2}$ e \\ César A. C. Marcondes ${ }^{3}$ \\ ${ }^{1}$ Departamento de Computação (DC) - Universidade Federal de São Carlos (UFSCar) \\ ${ }^{2}$ Departamento de Informática (DInf) - Instituto Federal do Tocantins (IFTO) \\ ${ }^{3}$ Divisão de Ciência da Computação (IEC) - Instituto Tecnológico de Aeronáutica (ITA) \\ \{douglas.godoy, jaime.souza\}@ufscar.br, emerson.barea@ifto.edu.br, cmarcondes@ita.br
}

\begin{abstract}
Resumo. É indicada a utilização de honeypots e honeynets para o estudo aprofundado do comportamento dos malwares para dispositivos de IoT, porém, alguns cuidados devem ser tomados garantindo que esses ambientes sejam capazes de detalhar corretamente todo ciclo de infecção dos malwares e não se tornem um ponto de origem de ataques às outras redes. Nessa linha, este trabalho apresenta o HonIoT, uma honeynet que suporta a inclusão de honeypots reais, emuladas ou virtualizadas, criando uma internet emulada que corresponde à parte atacada da rede, possibilitando o monitoramento completo de todo ciclo de infecção e propagação do malware. Testes preliminares apontaram boa capacidade de escalabilidade do ambiente e correta execução do fluxo de ações da honeynet.
\end{abstract}

\begin{abstract}
It is indicated the use of honeypots and honeynets for IoT devices malware behavior study in-depth, but some attention should be taken to ensure these environments are able to accurately detail every malware infection cycle and do not become a attacker to other networks. In this line, this work presents HonIoT, a honeynet that supports inclusion of real, emulated or virtualized honeypots, creating an emulated internet corresponding to the attacked part of the network, allowing the complete monitoring of the whole cycle of malware infection and propagation. Preliminary tests pointed to good environment scalability and correct execution of honeynet's stock flow.
\end{abstract}

\section{Introdução}

Nos últimos tempos foi perceptível o aumento da utilização de dispositivos de Internet das Coisas (Internet of Things - IoT) em áreas até então pouco exploradas. O desenvolvimento dessa estrutura tem sido bastante impulsionada principalmente pela evolução tecnológica de aparelhos de uso cotidiano, como telefones e TVs, porém, a IoT é mais ampla e atualmente está presente em diversas áreas, como indústrias, medicina, serviços, automobilismo, entre outras [Simpson et al. 2017] e [GARTNER 2017].

A ampliação da utilização de dispositivos de IoT veio acompanhada da necessidade de conectá-los às redes, privadas ou até mesmo pela internet, possibilitando sua utilização e gerenciamento remotos. Quanto a segurança, muitos desses dispositivos apresentam vulnerabilidades, que vão desde ausência de mecanismos de autenticação habilitados por padrão, passando por falhas em códigos, até problemas críticos de hardware [Dowling et al. 2017], 
que vêm sendo exploradas por métodos diversos [SPAMHAUS 2018] e com efeitos cada vez mais abrangentes e críticos [Bhunia and Gurusamy 2017] [Marzano et al. 2018].

O cenário apresentado aponta claramente que o aumento da utilização de dispositivos de IoT e o risco associado a esse tipo de ambiente são problemas relevantes de pesquisa. Uma abordagem bastante utilizada para coleta e análise de malwares são os honeypots e honeynets ([Sochor and Zuzcak 2014], [Koniaris et al. 2014] e [Pathan 2014]), porém, esses mesmos ambientes podem não ser capazes de capturar corretamente todo ciclo de infecção de um malware ou até passar a ser um ponto de origem de ataque às outras redes caso não tomados alguns cuidados [Agnaou et al. 2016] e [Cabaj et al. 2017].

Baseado no exposto, esse trabalho propõe uma arquitetura de honeynet modular com controle de propagação de malwares para dispositivos de IoT. Entre os destaques e aspectos mais relevantes, essa honeynet $(i)$ suporta a emulação de arquiteturas próprias de dispositivos de IoT com o uso de tecnologias de virtualização leve, lhe conferindo a possibilidade de ser livremente replicada em uma infraestrutura de computação genérica; (ii) emula a parte atacada da internet, portanto, não propagando ataques às outras redes enquanto fornece um ambiente propício para a completa infecção por parte do malware; e (iii) suporta o uso de equipamentos reais, emulados ou softwares de simulação como honeypot.

O restante desse trabalho está organizado da seguinte forma: a seção 2 apresenta os trabalhos relacionados ao tema principal da pesquisa. A seção 3 apresenta os requisitos a serem atendidos e os desafios impostos à proposta apresentada. Na seção 4 são apresentadas a arquitetura da honeynet e o detalhamento do protótipo desenvolvido. A seção 5 apresenta e comenta os resultados da avaliação do protótipo; e a seção 6 conclui e apresenta sugestões de trabalhos futuros.

\section{Trabalhos Relacionados}

São diversas as implementações de honeypots para dispositivos de IoT. Abaixo segue uma lista, não exautiva, de soluções divididas em algumas áreas de atuação.

O IoTPot [Pa et al. 2015] é um honeypot de baixa interatividade que emula banners reproduzidos artificialmente de diversos equipamentos de IoT utilizado para análise de tentativas de ataques por Telnet. Durante a fase de testes, os autores observaram mais de 76 mil tentativas de download de binários originados a partir de mais de 16 mil endereços IPs distintos. Adicionalmente, os autores fizeram o download de 43 exemplares de malwares para análise e constataram que eles estavam relacionados a 11 arquiteturas diferentes de CPU. Apesar dos resultados obtidos, os banners publicados pelo IoTPot são gerados manualmente, dificultando sua evolução e facilitando a identificação do honeypot pelo malware, já que não utiliza sistemas reais. Da mesma forma, ele não é capaz de detalhar o ciclo de infecção do malware apresentando as chamadas de rede e de sistemas nos alvos atacados.

Outra implementação existente é o HoneyThing [Erdem et al. 2018]. Honeypot de baixa interatividade que emula o protocolo TR-069, utilizado para gerenciamento de equipamentos de clientes (Customer-Premises equipament - $\mathrm{CPE}$ ), como modens e roteadores domésticos. Também possui o servidor Web RomPager $4.07^{1}$ simulando algumas

\footnotetext{
${ }^{1}$ https://www.allegrosoft.com/embedded-web-server-ae
} 
vulnerabilidades associadas ao protocolo TR-069 encontrados em modens. Apesar de ser um honeypot específico para CPEs, por ser de baixa interatividade, o HoneyThing apenas gera estatísticas de tentativas de acessos sem detalhar o processo de infecção do dispositivo, como também não fornece mecanismos de contenção em caso de comprometimento do honeypot.

Mais uma implementação, agora com foco em redes de sensores sem fio (Wireless Sensor Networks - WSN), é o ZigBee honeypot [Dowling et al. 2017], que reproduz um dispositivo com suporte a rede ZigBee para identificar ataques ao SSH. Os autores identificaram nos testes realizados que o tipo de ataque com maior ocorrência estava relacionado a DDoS. Assim como os outros honeypots apresentados, o ZigBee honeypot é de baixa interatividade, possuindo as mesmas limitações das soluções anteriores.

Por fim, outro exemplo de implementação é o HIoTPOT [Gandhi et al. 2018], que consiste em um honeypot de alta interatividade baseado no Raspberry PI conectado a duas redes, uma de dispositivos reais e a outra de dispositivos falsos. O HIoTPOT possui uma base de dados onde estão registrados todos usuários válidos e autenticados na rede. Quando um usuário não autenticado tenta acessar outro equipamento da rede, o honeypot automaticamente direciona seu tráfego para os dispositivos da rede falsa, mantendo segura a rede real enquanto possibilita a análise do processo de infecção do malware. Apesar de eficiente, o HIoTPOT tem custo elevado com a duplicação da estrutura da rede física, enquanto seu método de identificação de ataque é simples e passível de falha.

Como citado anteriormente, apesar de não exaustiva, a lista de honeypots apresentados indica que as soluções normalmente são baseados em serviços e protocolos específicos, reduzindo muito seu escopo e funcionalidades. Da mesma forma, não possuem mecanismos de contenção contra propagação de ataques caso o ambiente seja comprometido. Por outro lado, o exemplo de honeypot de alta interatividade apresentado é baseado em ambiente físico, elevando seu custo, além de possuir um mecanismo de contenção contra propagação de malwares muito simples e ineficiente.

\section{Requisitos e Desafios}

Ao propor o desenvolvimento de um honeypot de alta interatividade, ou honeynet, com controle de propagação de malwares para dispositivos de IoT emulados, alguns novos questionamentos surgem e levantam consigo requisitos ainda não contemplados.

Um honeypot de alta interatividade, assim como apresentado na Figura 1, tem como requisitos principais que as aplicações e serviços oferecidos por ele sejam reais, que possam ser comprometidos pelo malware e que o atacante tenha acesso irrestrito ao sistema operacional (S.O.) do dispositivo. Esses requisitos garantem que o malware não identifique que está em um honeypot, infectando completamente o ambiente e viabilizando a análise de seu funcionamento. Quanto aos desafios, são caracterizados pelo alto custo, dificuldade e complexidade de instalação e manutenção do ambiente, além do alto risco de comprometimento da estrutura e consequente propagação do malware utilizando o próprio honeypot como um ponto de ataque às outras redes [Hoepers et al. 2007].

Já os honeypots de baixa interatividade são reconhecidos por seu baixo custo de instalação, manutenção e risco de comprometimento, porém, como apresentado na seção 2, os resultados obtidos com sua utilização são limitados principalmente pelas deficiências 


\begin{tabular}{|l|c|c|}
\hline \multicolumn{1}{|c|}{ Características } & $\begin{array}{c}\text { Honeypot de baixa } \\
\text { interatividade }\end{array}$ & $\begin{array}{c}\text { Honeypot de alta } \\
\text { interativ./Honeynet }\end{array}$ \\
\hline Instalação & fácil & mais difícil \\
\hline Manutenção & fácil & trabalhosa \\
\hline Risco de comprometimento & baixo & alto \\
\hline Obtenção de informações & limitada & extensiva \\
\hline Necessidade de mecanismos de contenção & não & sim \\
\hline Atacante tem acesso ao S.O. real & não (em teoria) & sim \\
\hline Aplicações e serviços oferecidos & \multicolumn{2}{|c|}{ emulados e reais } \\
\hline Atacante pode comprometer o honeypot & não (em teoria) & sim \\
\hline
\end{tabular}

Figura 1. Características desejáveis ao honeynet com controle de propgação de malwares para dispositivos de loT. Fonte: adaptado [Hoepers et al. 2007].

de implementação ou ausência de recursos existentes nos sistemas reais, possibilitando serem descobertos pelos malwares mais avançados.

Baseado nas características apresentadas, identificamos que a proposta mais adequada para este trabalho é unir as características mais vantajosas dos dois tipos de honeypots (de baixa e alta interatividade), conforme destacado na Figura 1. Fazendo uso de sistemas reais de dispositivos de IoT em arquitetura correspondente emulada com o auxílio de softwares específicos, reduzindo o custo, simplificando a instalação e manutenção do ambiente; e emulando a parte atacada da rede com todas suas máquinas e serviços, impedindo a propagação do malware às outras redes enquanto cria um ambiente completo para infecção do malware, proporcionando meios para captura de informações para seu estudo.

\section{Materiais e Métodos}

Nesta seção são detalhados os elementos formadores da arquitetura da honeynet (seção 4.1), seguido da apresentação da estrutura física utilizada no desenvolvimento do protótipo HonIoT (seção 4.2) e do detalhamento do fluxo de ações e códigos do controlador do protótipo (seção 4.3).

\subsection{Arquitetura}

Para atingir os objetivos propostos, atendendo aos requisitos apresentados e solucionando os desafios impostos, foi definida uma arquitetura modularizada para honeynet de dispositivos de IoT. Essa arquitetura é responsável por suportar a análise de todo ciclo de infecção e propagação de malwares para IoT, com estrutura de honeypot de alta interatividade com controle de propagação, expondo totalmente um sistema de IoT aos ataques provindos da internet, enquanto monitora todo acesso e fluxo de dados num ambiente controlado, criando a representação de parte da internet quando o honeypot passa a ser um ponto de origem e disseminação de ataques.

Para facilitar a compreensão de sua estrutura e o funcionamento de cada componente, a arquitetura foi dividida em quatro módulos principais, conforme apresentado na Figura 2. Abaixo estão descritas as funcionalidades dos elementos pertencentes a cada módulo e a relação entre eles: 
Dispositivos de IoT: corresponde aos dispositivos destinados a sofrerem ataques na honeynet. Suporta a utilização de honeypots de baixa interatividade, com reprodução de serviços ou sistemas de IoT virtualizados em hardware genérico ou com a utilização de plataformas e sistemas construídos especialmente para capturar informações de acessos maliciosos; ou alta interatividade, suportando a utilização de sistemas e dispositivos de IoT emulados em plataformas específicas ou reais conectados à honeynet.

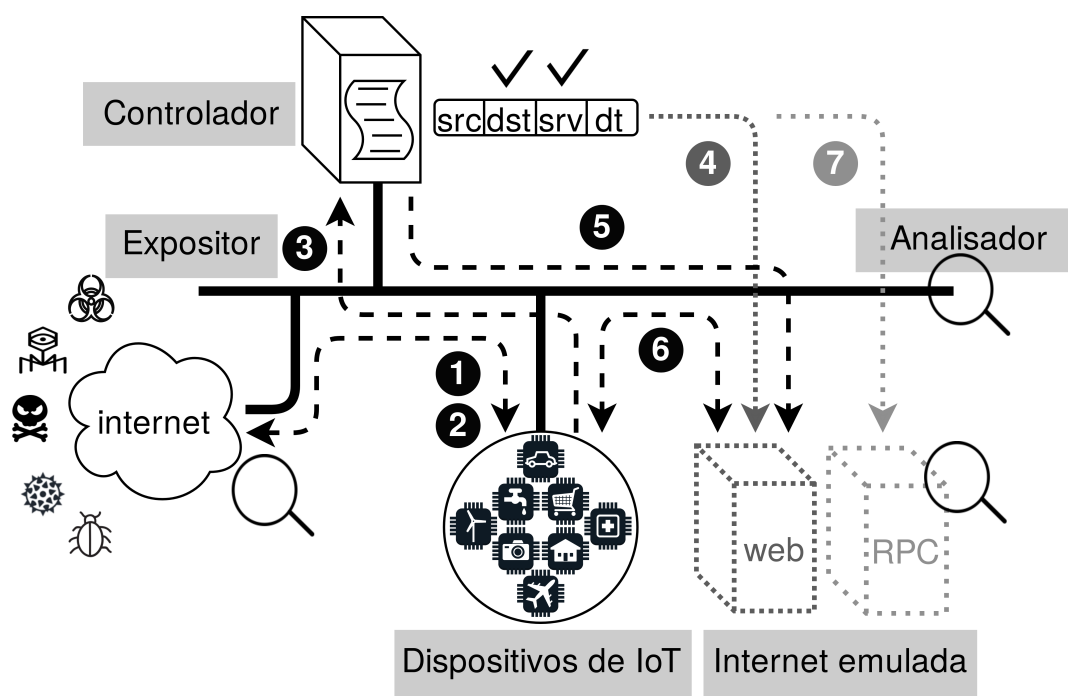

Figura 2. HonloT: arquitetura da honeynet com controle de propagação de malwares para dispositivos loT.

Outra função desse módulo é garantir que o malware não identifique que está em um honeypot, possibilitando a infecção completa do ambiente. Alguns malwares utilizamse de artifícios variados para identificar se o ambiente é um honeypot, limintando ou até mesmo anulando seu comportamento quando a identificação é positiva. Dessa forma, é aconselhável utilizar plataformas e sistemas que reproduzam fielmente a original, com o auxílio de emulação da arquitetura correta e configurações adicionais necessárias, como a implementação de banners que correspondam aos dos serviços reais e a ocultação de parâmetros e informações do sistema base de emulação do ambiente.

Expositor: esse módulo corresponde a toda infraestrutura de rede necessária para exposição completa dos dispositivos de IoT (honeypot). Sua função é possibilitar que malwares provindos da internet acessem o honeypot para infectá-lo completamente. Essa fase corresponde aos Fluxos 1 e 2 apresentados na Figura 2 (fluxos identificados pelas bolas pretas com os números 1 e 2). No Fluxo 1, o honeypot é acessado livremente por qualquer malware com origem na internet, sem restrição alguma, possibilitando sua exposição completa e, consequentemente, aumentando a probabilidade de captura de informações de malwares diversos. Já o Fluxo 2 permite que o honeypot acesse os servidores de comando e controle $(\mathrm{C} \& \mathrm{C})$ dos malwares distribuídos na internet, e vice-versa, a fim de completar o ciclo de infecção do malware.

Toda a exposição representada pelos Fluxos 1 e 2 na arquitetura são gerenciadas pelo Controlador da honeynet, módulo detalhado em seguida.

Controlador: esse módulo é considerado o mais importante da honeynet, pois, é ele que garante a inteligência da rede atuando como um gerenciador de tráfego pró- 
ativo, durante a infecção do honeypot; e também reativo ao comportamento do malware. Inicialmente sua função é permitir o fluxo de dados entre os malwares provindos da internet e o honeypot, como apresentado anteriormente no módulo Expositor, onde toda comunicação é baseada em regras de controle de tráfego e definição de rotas que permitem o encaminhamento dos dados entre os dispositivos envolvidos na comunicação.

A partir da infecção completa do honeypot, todo tráfego originado por ele deve ser interceptado pelo Expositor e direcionado ao Controlador (Fluxo 3), pois, a partir desse momento, os novos fluxos podem estar relacionados aos procedimentos de propagação e disseminação do malware, que devem ser evitados para não tornar a honeynet num ponto de origem de ataques. Ao mesmo tempo, a análise dessas chamadas de rede são material fundamental para o estudo e entendimento do comportamento e funcionamento do malware.

Com o tráfego interceptado, o Controlador identifica e extrai os parâmetros de endereçamento de rede e do serviço solicitados das máquinas envolvidas na comunicação - endereço IP de destino, protocolo e portas de serviço utilizadas. Com posse dessas informações, o Controlador tem a função de criar as máquinas da Internet emulada sob demanda, criando automaticamente máquinas clone configuradas com os parâmetros de rede e de serviços previamente extraídos pelo Controlador (Fluxo 4).

A partir da criação da máquina clone, o controlador cria as regras de roteamento e direcionamento de tráfego, instalando-as no Expositor para então encaminhar os dados da solicitação de comunicação diretamente à máquina clone na Internet emulada (Fluxo 5). A partir desse momento, toda nova comunicação entre o honeypot e a máquina clone é feita diretamente através do ambiente Expositor (Fluxo 6), dessa forma, nenhum tráfego originado no honeypot correspondente a uma tentativa de propagação do malware, ou qualquer outra ação dele, será propagada para outras redes. Esse procedimento impede que a honeynet seja um ponto de ataque e viabiliza o monitoramento do tráfego de rede originado pelo malware, possibilitando sua análise detalhada. A cada solicitação de comunicação iniciada pelo honeypot a um novo destino, protocolo ou serviço, esse processo é repetido (Fluxo 7).

Analisador: esse módulo é responsável por capturar dados que servirão como base de informações para a análise de todo processo de infecção, comprometimento e propagação do malware. As ferramentas pertencentes a esse módulo estão distribuídas em quatro áreas específicas da arquitetura:

- Internet: aqui o analisador monitora todas as tentativas de acesso ao honeypot, incluindo tempo, volumetria de tráfego, origem dos ataques e quais mecanismos são utilizados.

- Dispositivos de IoT: o monitoramento interno nos dispositivos de IoT provê informações que podem ser utilizadas para análise das ações do malware nesses dispositivos, através de monitoramento da utilização de memória, processos criados, chamadas de sistema ou outros parâmetros que se fizerem necessários. Vale ressaltar que o monitoramento nesse ponto dependente do tipo de honeypot utilizado, pois, devem ser respeitadas restrições de acesso a determinados parâmetros por questão de licenças ou limitações técnicas em equipamentos ou softwares proprietários.

- Rede da Internet emulada: monitora todo tráfego de rede passante entre o honeypot 
e todos elementos da Internet emulada, assim como realizado no link de Internet. Monitora o fluxo de dados correspondente à propagação do malware a partir do comprometimento do honeypot.

- Máquinas da Internet emulada: aqui o analisador monitora as ações executadas pelo malware internamente nas máquinas da Internet emulada, como apresentado no Dispositivo de IoT. O objetivo é gerar informações que sirvam como base para análise do comportamento do malware nas máquinas atacadas durante sua propagação.

Com a utilização de todas informações obtidas no módulo Analisador, é possível realizar um estudo aprofundado de todo ciclo de infecção de um malware.

\subsection{HonIoT}

Para validar a arquitetura da honeynet com controle de propagação de malwares para dispositivos de IoT proposta neste trabalho e apresentada na subseção 4.1, foi desenvolvido o protótipo HonIoT (Honeynet IoT), cuja finalidade é simular parte do processo de infecção sofrido por um honeypot de alta interatividade a partir de malwares originados na internet, possibilitando o estudo dos mecanismos de infecção e propagação.

O HonIoT é um protótipo que une um conjunto de aplicações de rede e emulação leve pré-existentes, a elementos desenvolvidos especialmente para esse fim. De modo geral, baseia-se na plataforma de emulação Qemu versão 2.11.1 como base para o Dispositivo de IoT (honeypot); o OVS versão 2.9.2 e Wireshark versão 2.6.5 na estrutura do Expositor; o LXD versão 3.0.3 como base para as máquinas da Internet emulada; e o Ryu versão 4.24 como controlador para SDN do OVS.

A estrutura física do HonIoT é composta por dois servidores físicos, cada um contendo um processador de 3.50GHz e 8 núcleos, 16GB de memória RAM e múltiplas interfaces de rede de $1 \mathrm{Gbps}$ cada. Além de um computador com processador de $3 \mathrm{GHz}$ e 4 núcleos, 2 GB de memória RAM e duas interfaces de rede de $1 \mathrm{Gbps}$ cada. As máquinas foram conectadas fisicamente conforme apresentado na Figura 3. Em todas as máquinas foi instalado o S.O. Ubuntu 16.04.4 LTS Server sem serviços adicionais, propiciando um ambiente livre de interferências indesejáveis.

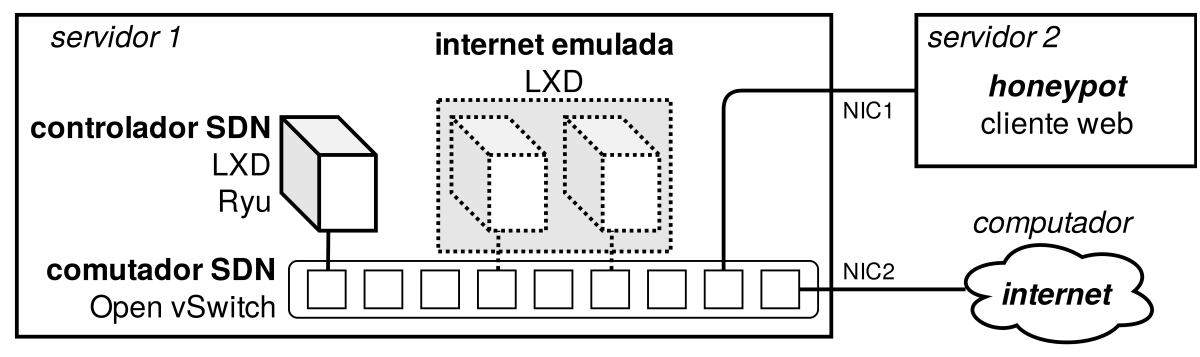

Figura 3. Topologia para o protótipo HonloT.

Conforme apresentado na Figura 3, o servidor 1 hospeda todo ambiente de controle e emulação responsáveis por reproduzir a porção da rede atacada. Tal ambiente é composto por controlador SDN (container LXD com Ryu instalado), comutador SDN (OVS) e internet emulada (containers LXD criados sob demanda). O servidor 2 possui o S.O. Linux instalado contendo softwares de servidor e cliente Web instalados sobre 
uma arquitetura ARM emulada (Qemu) simulando um honeypot. Já o computador que representa a internet possui apenas o S.O. Linux instalado diretamente em seu hardware.

O servidor 1 possui uma interface de rede conecta diretamente ao servidor 2 . Essa interface também está conectada logicamente ao comutador $S D N$, possibilitando a comunicação entre o ambiente virtualizado da honeynet e o honeypot emulado. $\mathrm{O}$ servidor 1 também possui uma interface de rede conectada fisicamente ao computador internet $\mathrm{e}$ logicamente ao comutador $S D N$, possibilitando a interligação de todos elementos virtuais e físicos no protótipo.

\subsection{Fluxo de Ações}

Quanto ao fluxo das ações executadas para validação do protótipo, inicialmente foram geradas requisições de rede com origem na internet e destinadas ao servidor Web do honeypot, simulando a infecção por um malware. Essas chamadas são encaminhadas sem restrição no comutador $S D N$, ao mesmo tempo em que o tráfego de rede é monitorado ao passar pelo comutador. Essa etapa da validação do protótipo corresponde ao Fluxo 1 da Figura 2.

Para isso, assim que percebe o fluxo de dados, o comutador $S D N$ intercepta e direciona os pacotes ao controlador $S D N$, que inspeciona o parâmetro correspondente ao tipo de protocolo da camada 4 , identificando se o segmento é TCP (bits $==2$ indica que o segmento possui a flag $S Y N$ utilizada). A partir dai, o controlador $S D N$ extrai os endereços IP de origem e destino do datagrama identificando se a origem está na internet (src_port) e o destino é o honeypot (HONEY POT_IP). Caso a comunicação atenda essa premissa, o controlador $S D N$ cria a regra que permite a comunicação entre a máquina da internet e o honeypot (match1) e a instala no comutador $S D N$ (add_flow) (Figura 4).

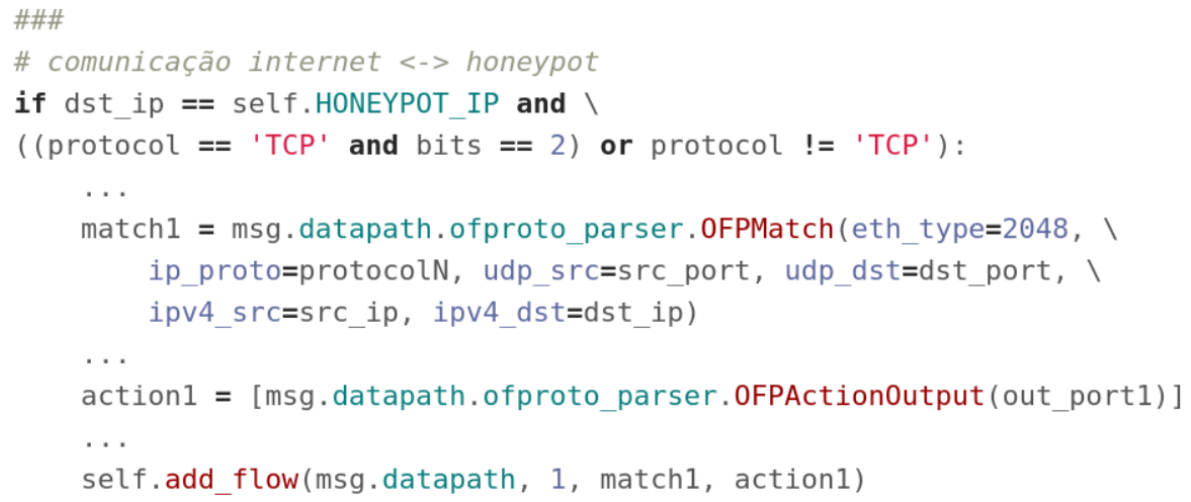

Figura 4. Código Python do controlador que permite máquinas da internet acessarem livremente o honeypot.

Seguindo esse processo, quando o honeypot inicia a comunicação com um servidor de C\&C, o tráfego também deve ser permitido, conforme descrito na seção 4.1, pois essa comunicação é considerada requisito para que o malware continue o processo de infecção. Durante esse processo, o controlador $S D N$ verifica se o endereço IP de destino contido no datagrama interceptado (dst_ip) existe na white_list (ip_list) recuperada em [Emerging Threats 2017]. Essa filtragem por endereços da white_list permite que o 
controlador identifique quais endereços IP são de servidores de $\mathrm{C} \& \mathrm{C}$, permitindo que o controlador $S D N$ crie regras liberando o tráfego para esses servidores (Figura 5).

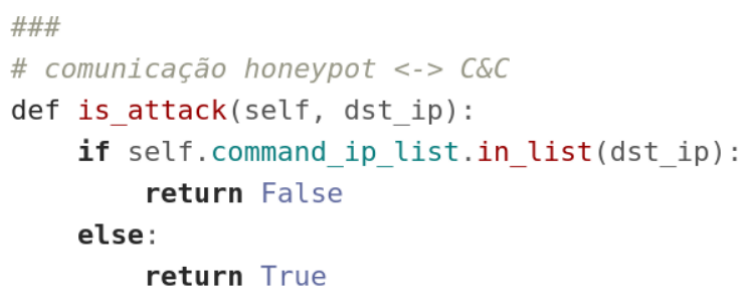

Figura 5. Código Python do controlador que permite a comunicação do honeypot com servidores de C\&C.

Todo tráfego gerado no honeypot com destino a qualquer endereço IP não constante na white list pode representar uma tentativa de propagação do malware que deve ser impedida pelo HonIoT. Para isso, o comutador $S D N$ intercepta o fluxo de dados e o encaminha ao controlador $S D N$ que, por sua vez, extrai as informações de endereçamento das máquinas, protocolos e serviços contidos no fluxo, e acessa o gerenciador de containers localizado no servidor 1 através de uma conexão SSH, solicitando a criação de um novo container LXD a partir de modelos pré-estabelecidos com base no serviço desejado (DEFAULT_CONTAINER_TEMPLATE) (Figura 6). A partir desse momento, o gerenciador de containers configura o container recém criado com os parâmetros de rede identificados anteriormente.

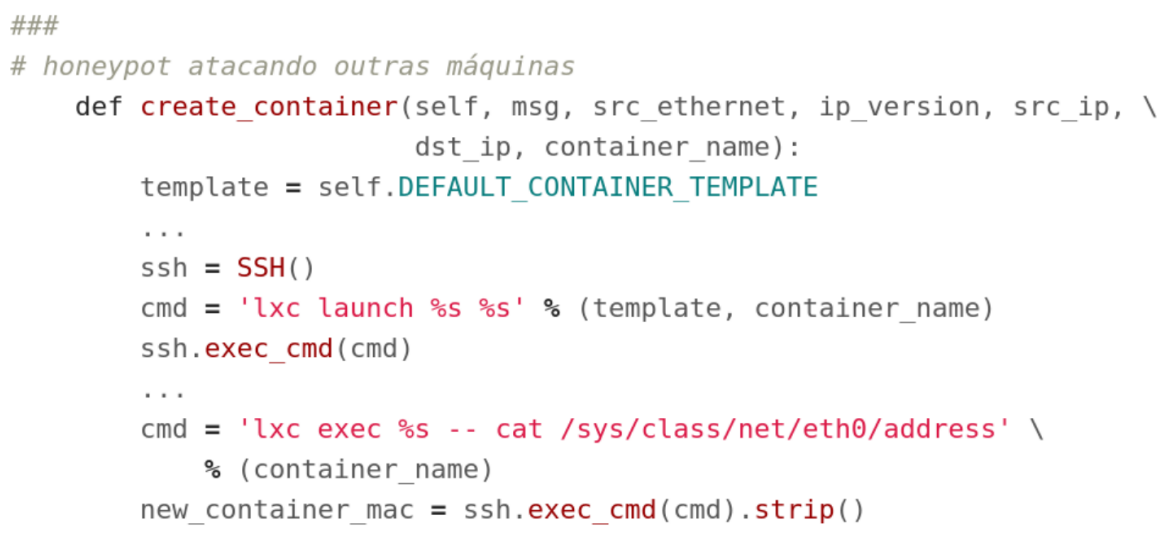

Figura 6. Exemplo de código do controlador que cria containers na Internet emulada.

Feito isso, o controlador $S D N$ recupera a informação de endereço de camada 2 (Media Access Control - MAC) atribuído automaticamente ao container recém gerado pelo serviço LXD (new_container_mac), cria e instala uma regra (action 1 e action 2 Figura 7) no comutador SDN permitindo o tráfego entre o honeypot e o container com base nesse endereço, possibilitando assim o encaminhamento de tráfego diretamente entre essas máquinas. 


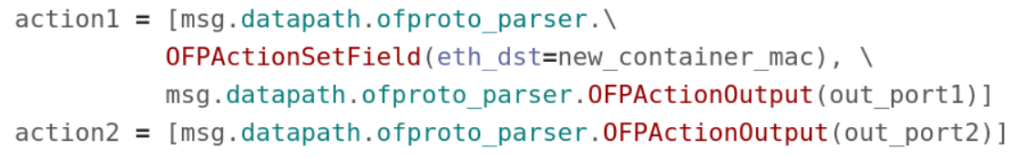

\section{Figura 7. Código Python do controlador que cria e instala a regra para comuni- cação com o container da internet emulada.}

Todo esse processo é repetido sempre que o honeypot gera fluxos de dados para novos destinos, protocolos ou serviços, impedindo que o honeypot propague o malware, além de viabilizar a análise de todo esse processo com o monitoramento dos fluxos de dados na rede e as ações executadas pelo malware nas máquinas da internet emulada.

\section{Testes de Validação e Discussão dos Resultados}

Para avaliar o completo funcionamento do protótipo HonIoT, dividimos os testes em 4 etapas principais que estão detalhadas a seguir:

Experimentos de Invasão ao honeypot: esse teste analisou o correto funcionamento do processo em que máquinas distintas da internet acessam serviços disponíveis no honeypot, simulando uma tentativa de iniciar a infecção. Para isso, foram geradas 100 requisições ao serviço Web partindo de IPs gerados aleatoriamente na máquina da internet destinadas ao honeypot. Nesse teste, $100 \%$ das requisições foram devidamente tratadas pelo comutador $S D N$ e controlador $S D N$, seguindo o procedimento apresentado na seção 4.3, Figura 4, com todas solicitações de acesso devidamente encaminhadas ao honeypot, conforme demonstraram os rastros de execução. Essa validação foi considerada satisfatória.

Experimentos de Comunicação Honeypot e Servidores de C\&C: em seguida, um segundo experimento foi o de acompanhar o correto funcionamento da comunicação livre do honeypot com servidores de $\mathrm{C} \& \mathrm{C}$ a qualquer momento. Esse acesso deve ser permitido quando o endereço IP de destino do datagrama emitido pelo honeypot constar na white list de servidores de C\&C. Ao invés de testar com um malware imprevisível, foram feitos testes de conexão iniciadas a partir do servidor 2 (honeypot), para isso, foram gerados requisições Web originadas no honeypot e destinadas aos endereços IP dos servidores constantes na white list de servidores de C\&C. Ao final do teste, observou-se que todas requisições foram devidamente tratadas pelo comutador $S D N$ e controlador $S D N$, e encaminhadas para a saída de internet corretamente. Os rastros de execução demonstraram que esse teste de validação foi considerado satisfatório.

Experimentos de Criação das máquinas na Internet emulada: esse terceiro experimento teve o propósito de verificar se o controlador $S D N$ solicita corretamente a criação de um ou mais containers ao Servidor 1 após receber datagramas IP com parâmetros de rede "não constantes" em regras já instaladas no comutador $S D N$; e se os containers são criados e inicializados corretamente para encaminhamento do tráfego partindo do honeypot em tempo hábil.

Nessa etapa, o interesse era em dois cenários possíveis. O primeiro verificou o comportamento do ambiente, na forma dessa implementação, quando solicitadas chamadas de grupos de containers de forma sequencial, onde um container posterior somente é solicitado quando o anterior já está totalmente funcional. A ideia era estudar o comportamento 
de um malware que interage e analisa o ambiente em que está antes de tomar a próxima decisão.

O segundo cenário analisou o comportamento do ambiente quando grupos de containers são solicitados em rajadas pelo honeypot, simulando uma situação de ocorrência de scan de rede. O scan de rede pode ser intenso e requerer a criação de dezenas ou centenas de containers em cada execução. Esse cenário permitiu verificar e analisar a escalabilidade e estabilidade do protótipo nesse tipo de situação.

Os resultados demonstrados na Figura 8 apresentam a utilização de memória (em MB) agrupada por conjuntos de containers criados sequencialmente, ou seja, no cenário 1. Os dados mostram que a utilização de memória está diretamente relacionada ao número de containers criados, porém, seu crescimento não é linearmente perfeito, partindo de aproximados $70 \mathrm{MB}$ para criação de 1 container, passando por aproximados $463 \mathrm{MB}$ de memória consumida para um grupo de 50 containers, chegando a até $925 \mathrm{MB}$ de memória consumida para grupos de 100 containers, sempre com 100\% de sucesso na criação dos containers. Os resultados indicam uma sobrecarga de memória ao serem criados mais containers. Não foi investigado a fundo o porque desse comportamento, mas pode ser em função de replicação de dados do sistema operacional.

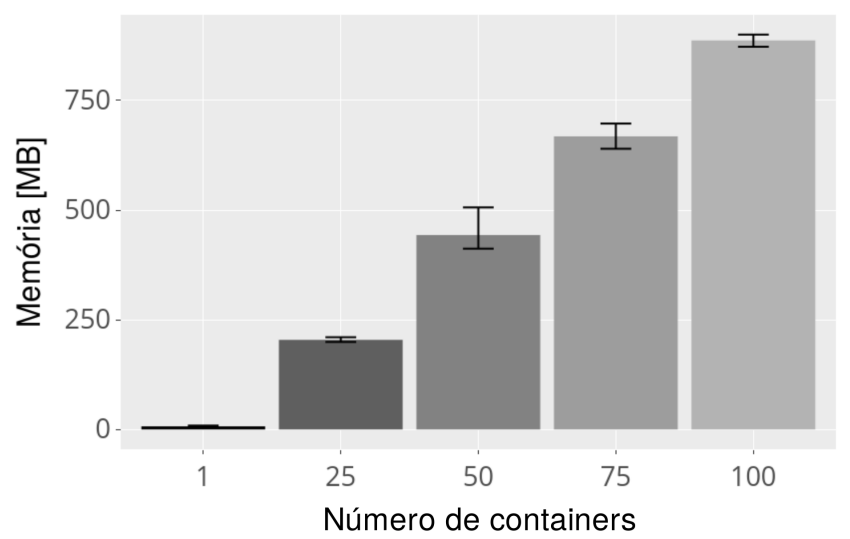

Figura 8. Memória (em MB) consumida para criação dos containers correspondentes às máquinas da Internet emulada.

Apesar desse comportamento de sobrecarga adicional por container adicional, o protótipo comprova que a utilização de containers LXD na Internet emulada é promissora quando considerado o consumo total de memória versus o número de máquinas emuladas. Ou seja, 100 containers em uma máquina servidora pode ser considerado baixo uso de recursos.

Outro resultado importante identificado é o comportamento padrão quanto ao tempo de criação dos containers, que foi de 4.1 segundos em média por container. Esse tempo tem o revés de ser elevado para tratar o encaminhamento de um pacote, podendo gerar desconfiança em um malware mais sofisticado sobre a possibilidade de ele estar em um honeypot.

Com relação ao cenário 2, onde é analisado o comportamento do protótipo frente a situações de rajadas de pacotes, foram verificados o tempo de criação para os grupos 
de containers e a quantidade de sucesso nesse procedimento. Como não existe tempo mínimo entre as chegadas de pacotes, o controlador $S D N$ recebe múltiplas requisições simultâneas por meio de eventos em threads, e repassa para o gerenciador de containers as solicitações de criação no melhor esforço da tecnologia utilizada no protótipo.

Os dados da Figura 9a apresentam que, apesar das solicitações terem sido geradas em rajadas a partir do honeypot, onde verificou-se um tempo médio de apenas 0,6 segundos para o encaminhamento de $100 \%$ das solicitações, houve um aumento considerável de tempo para criação dos containers na Internet emulada do servidor 1, chegando a aproximadamente 53 segundos em média para grupos de 30 containers solicitados. Esse comportamento ocorre devido os recursos de criação de containers serem compartilhados e a contenção ser grande.

Além dos tempos de instanciação serem elevados, outro dado importante é a grande variação do tempo obtido entre os testes, que demonstrou instabilidade do ambiente do protótipo nesse caso.

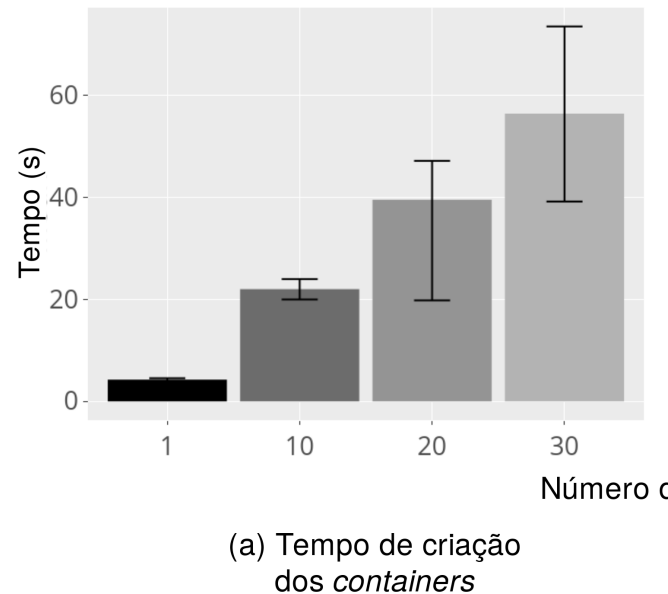
dos containers

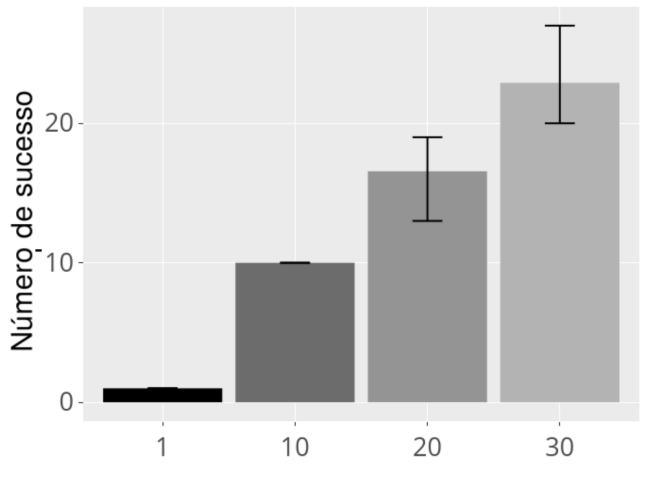

(b) Número de containers criados com sucesso

Figura 9. Tempo (segundos) consumidos para criação de grupos de containers solicitados em rajadas (a) e quantidade de containers criados com sucesso por grupos de containers solicitados em rajadas (b).

Para analisar essa questão de instabilidade e a incapacidade de dar vazão rápida para um número grande de instanciação de containers, uma observação importante é o aumento expressivo da taxa de insucesso com o aumento da quantidade de containers solicitados nas rajadas. Conforme apresentado na Figura 9b, a taxa de insucesso é de aproximadamente $10 \%$ a partir da solicitação de criação de 20 containers, aumentando consideravelmente para até $20 \%$ de falhas para grupos de 30 containers solicitados.

Análises preliminares dos resultados apontam que as tecnologias utilizadas no protótipo possuem deficiência na forma como o controlador conecta no servidor 1 para solicitar a criação dos containers, utilizando conexões SSH através do Python Paramiko. Os rastros da execução apontaram erros no estabelecimento de conexões entre essas duas máquinas quando as solicitações chegam em rajadas. Uma possível alternativa é a utilização de sockets Unix ou outro tipo de comunicação de rede menos complexa que uma conexão SSH. Uma outra opção é a pré-instanciação de centenas de containers no inicio da criação do ambiente experimental, cabendo ao controlador somente a tarefa de 
configurá-los com os parâmetros de rede extraídos previamente durante a interceptação dos fluxos de dados.

Comunicação com as máquinas da Internet emulada: o último experimento de validação verificou o funcionamento do encaminhamento do tráfego partindo do honeypot e destinado aos containers da Internet emulada, ao invés de direcioná-lo para saída pelo link de internet real da honeynet. Nessa etapa, basicamente após a criação dos containers na Internet emulada, foi observado a criação as regras de encaminhamento no comutador $S D N$, atualizando a tabela de fluxos e, a partir dai, todo tráfego partindo do honeynet foi encaminhando corretamente, sem atrasos relevantes ou quebra de conexão TCP para as partes correspondentes. Os rastros de execução do controlador e dos containers envolvidos demonstraram que esse teste de validação foi considerado satisfatório.

\section{Conclusões e Trabalhos Futuros}

Este trabalho apresentou o HonIoT, uma honeynet para dispositivos de IoT que suporta honeypots baseados em equipamentos físicos e reais, simulados ou emulados. Garante a exposição completa do honeypot aos malwares disponíveis na internet, enquanto garante o controle de propagação do malware criando uma internet emulada e protegida, além de propiciar um ambiente completo de monitoramento das ações do malware através do monitoramento de suas ações no honeypot, na rede e nas máquinas emuladas.

Testes preliminares demonstraram bom nível de escalabilidade da honeynet quanto ao tempo de resposta às solicitações de criação de máquinas na rede emulada e quantidade suportada, bem como o correto funcionamento do fluxo de ações do protótipo proposto.

Como trabalhos futuros, considera-se relevante investigar maneiras mais sofisticadas para identificar quando o malware que infectou o honeypot pretende acessar servidores de $\mathrm{C} \& \mathrm{C}$ ou realizar um ataque, testar outras técnicas de conexão entre o controlador SDN e a máquina hospedeira da Internet emulada para melhorar a taxa de sucesso da criação de containers em situação de rajadas de fluxos de rede. Outra necessidade é testar o HonIoT em ambiente real para monitorar seu comportamento em produção.

\section{Agradecimentos}

Este trabalho teve apoio da Coordenação de Aperfeiçoamento de Pessoal de Nível Superior - Brasil (CAPES) - Código de Financiamento 001 e do Instituto Federal do Tocantins (IFTO).

\section{Referências}

Agnaou, A., Kalam, A. A. E., Ouahman, A. A., and Montfort, M. D. (2016). Reduce positive and negative falses from attacks collected from the deployment of distributed honeypot network. CoRR, abs/1611.03252.

Bhunia, S. S. and Gurusamy, M. (2017). Dynamic attack detection and mitigation in iot using sdn. In 2017 27th International Telecommunication Networks and Applications Conference (ITNAC), pages 1-6.

Cabaj, K., Gawkowski, P., Grochowski, K., Nowikowski, A., and Żórawski, P. (2017). The impact of malware evolution on the analysis methods and infrastructure. In 2017 Federated Conference on Computer Science and Information Systems (FedCSIS), pages 549-553. 
Dowling, S., Schukat, M., and Melvin, H. (2017). Data-centric framework for adaptive smart city honeynets. In 2017 Smart City Symposium Prague (SCSP), pages 1-7.

Dowling, S., Schukat, M., and Melvin, H. (2017). A zigbee honeypot to assess iot cyberattack behaviour. In 2017 28th Irish Signals and Systems Conference (ISSC), pages $1-6$.

Emerging Threats (2017). Emerging threats botnet command and control drop rules. http://rules.emergingthreats.net/open/suricata/rules/ botcc.rules. Acessado em: 18/12/2017.

Erdem, O., Pektas, A., and Kara, M. (2018). Honeything: A new honeypot design for cpe devices. KSII Transactions on Internet and Information Systems, 12:4512-4526.

Gandhi, U. D., Kumar, P. M., Varatharajan, R., Manogaran, G., Sundarasekar, R., and Kadu, S. (2018). Hiotpot: Surveillance on iot devices against recent threats. Wireless Personal Communications, 103(2):1179-1194.

GARTNER (2017). 6.4 billion connected "things"will be in use in 2016. https : / / www . gartner.com/newsroom/id/3165317. Acessado em: 13/04/2017.

Hoepers, C., Steding-Jessen, K., and Chaves, M. H. P. C. (2007). Honeypots e honeynets: Definições e aplicações. https : / / www . cert.br/docs/whitepapers / honeypots-honeynets/. Acessado em: 18/12/2018.

Koniaris, I., Papadimitriou, G., Nicopolitidis, P., and Obaidat, M. (2014). Honeypots deployment for the analysis and visualization of malware activity and malicious connections. In 2014 IEEE International Conference on Communications (ICC), pages $1819-1824$.

Marzano, A., Alexander, D., Fazzion, E., Fonseca, O., Cunha, Italo e Hoepers, C., StedingJessen, K., e Chaves, M. H. P. C., Guedes, D., and Meira Jr., W. (2018). Monitoramento e caracterização de botnets bashlite em dispositivos iot. Simpósio Brasileiro de Redes de Computadores (SBRC), 36.

Pa, Y. M. P., Suzuki, S., Yoshioka, K., Matsumoto, T., Kasama, T., and Rossow, C. (2015). Iotpot: Analysing the rise of iot compromises. In 9th USENIX Workshop on Offensive Technologies (WOOT 15), Washington, D.C. USENIX Association.

Pathan, A.-S. K. (2014). The State of the Art in Intrusion Prevention and Detection. Auerbach Publications, Boston, MA, USA.

Simpson, A. K., Roesner, F., and Kohno, T. (2017). Securing vulnerable home iot devices with an in-hub security manager. In Pervasive Computing and Communications Workshops (PerCom Workshops), 2017 IEEE International Conference on, pages 551556. IEEE.

Sochor, T. and Zuzcak, M. (2014). Study of internet threats and attack methods using honeypots and honeynets. In Kwiecień, A., Gaj, P., and Stera, P., editors, Computer Networks, pages 118-127, Cham. Springer International Publishing.

SPAMHAUS (2018). Spamhaus botnet threat report 2017. https : / /www . spamhaus . org/news/article/772/spamhaus-botnet-threat-report-2017. Acessado em: 05/03/2018. 